\title{
PERFIL E EVITABILIDADE DE ÓBITO NEONATAL EM UM MUNICÍPIO DA AMAZÔNIA LEGAL
}

\author{
Kátia Fernanda Alves Moreira1 ${ }^{1}$ Bianca Oyola Bicalho², Luanna Cristina Sales Santos², Fabíola Mara \\ Gonçalves de Siqueira Amaral ${ }^{3}$, Nathalia Halax Orfão ${ }^{4}$, Mônica Pereira Lima Cunha ${ }^{5}$
}

\begin{abstract}
RESUMO: Descrever o perfil dos óbitos neonatais e causas evitáveis em Porto Velho - Rondônia, no período de 2011 a 2015. Estudo descritivo, do tipo transversal, realizado com dados do Sistema de Informação de Nascidos Vivos e Sistema de Informação de Mortalidade. Foram elegíveis 279 óbitos, sendo 208 (74,55\%) precoce, 156 $(55,91 \%)$ sexo masculino, $194(69,53 \%)$ peso até 2.499 gramas, Apgar variando de 6 a 10 no $1^{\circ} 170(60,93 \%)$ e $5^{\circ}$ minuto $226(81 \%)$, sem anomalia 261 (93,55\%). O coeficiente de mortalidade neonatal diminuiu nos anos de $2014(3,79)$ e $2015(2,28)$. Quanto às causas básicas, 87 (31,18\%) eram reduzíveis por adequada atenção ao recém-nascido, seguido de $47(16,85 \%)$ por adequada atenção à mulher na gestação. A alta prevalência desses óbitos alerta para necessidade de melhoria na assistência pré-natal e estruturação da rede cegonha, para garantir atenção de qualidade, integrada e contínua ao binômio mãe/filho.
\end{abstract}

DESCRITORES: Recém-nascido; Mortalidade infantil; Saúde Pública; Causas de Morte.

\section{PROFILE AND PREVENTABILITY OF NEONATAL DEATHS IN A CITY IN LEGAL AMAZON}

ABSTRACT: Describe the profile of neonatal deaths and preventable causes in Porto Velho, state of Rondônia, in the period from 2011 to 2015. It is a descriptive, cross-sectional study conducted with data from the Sistema de Informação de Nascidos Vivos (Live Births Information System) and Sistema de Informação de Mortalidade (Mortality Information System). Two-hundred and seventy-nine deaths were eligible, with 208 (74.55\%) premature births, 156 (55.91\%) male, 194 (69.54\%) weighing up to 2,499 grams, with Apgar values ranging from 6 to 10 in the first minute $(170 ; 60.93 \%)$ and fifth minute $(226 ; 81 \%)$; and 261 without anomalies $(93.55 \%)$. The neonatal mortality coefficient decreased in years 2014 (3.79) and 2015 (2.28). Regarding basic causes, 87 (31.18\%) were reducible through adequate attention to newborns, followed by $47(16.85 \%)$ reducible through adequate attention to women during pregnancy. The high prevalence of these deaths calls attention to the need to improve prenatal care and the structure of the Rede Cegonha (Stork Network) in order to ensure care that has quality and is integrated and continuous for mother and children.

DESCRIPTORS: Newborn; Child Mortality; Public Health; Cause of Death.

\section{PERFIL Y EVITABILIDAD DE DECESO NEONATAL EN UN MUNICIPIO DE LA AMAZONIA LEGAL}

RESUMEN: Describir el perfil de los decesos neonatales y causas evitables en Porto Velho - Rondônia, en el período de 2011 a 2015. Estudio descriptivo, del tipo transversal, realizado con datos del Sistema de Información de Nacidos Vivos y Sistema de Información de Mortalidad. Fueron elegibles 279 decesos, siendo 208 (74,55\%) precoces, 156 (55,91\%) de sexo masculino, 194 (69,53\%) con peso hasta 2.499 gramos, Apgar variando de 6 a 10 en $1^{\circ}$ minuto $170(60,93 \%)$ y en $5^{\circ} 226(81 \%)$, sin anomalías 261 (93,55\%). El coeficiente de mortalidad neonatal disminuyó en $2014(3,79)$ y $2015(2,28)$. Respecto de las causas básicas, $87(31,18 \%)$ eran reducibles mediante adecuada atención del recién nacido, seguida de 47 (16,85\%) por adecuada atención de la mujer embarazada. La alta prevalencia de estos decesos alerta sobre necesidad de mejora de atención prenatal y estructuración de una red cigüeña, garantizando atención calificada, integrada y continua al binomio madre/hijo.

DESCRIPTORES: Recién Nacido; Mortalidad Infantil; Salud Pública; Causas de Muerte.

${ }^{1}$ Enfermeira. Doutora em Enfermagem. Docente de Enfermagem da Fundação Universidade Federal de Rondônia. Porto Velho, RO, Brasil.

${ }^{2}$ Discente de Enfermagem. Fundação Universidade Federal de Rondônia. Porto Velho, RO, Brasil.

${ }^{3}$ Enfermeira. Mestranda em Ensino em Ciências da Saúde. Docente de Enfermagem da Fundação Universidade Federal de Rondônia. Porto Velho, RO, Brasil.

${ }^{4}$ Enfermeira. Doutora em Enfermagem. Docente de Enfermagem da Fundação Universidade Federal de Rondônia. Porto Velho, RO, Brasil.

${ }^{5}$ Enfermeira. Doutoranda em Ciências da Saúde. Docente de Enfermagem da Fundação Universidade Federal de Rondônia. Porto Velho, RO, Brasil. 


\section{- INTRODUÇÃO}

A taxa de mortalidade infantil (TMI) é um dos indicadores de saúde utilizados para avaliar as condições de saúde, desenvolvimento econômico e a qualidade de vida de uma população(1). Globalmente, quase $40 \%$ da mortalidade infantil (MI) está relacionada a causas infecciosas evitáveis ou tratáveis, como pneumonia, doenças diarreicas e complicações no nascimento e desnutrição ${ }^{(2)}$.

Em 2015, das 5,9 milhões de mortes em menores de 5 anos, 2,7 milhões ocorreram no período neonatal. No Brasil, mesmo com a redução de 73\% da TMI, 16 óbitos infantis ocorrem para cada 1.000 nascidos vivos (NV), podendo sofrer variações, em virtude das disparidades regionais. Em regiões mais pobres, tais como Nordeste e Norte, a Taxa de Mortalidade Neonatal (TMN) apresenta elevados percentuais em decorrência da precariedade das condições sanitárias, acesso e uso dos serviços de saúde, e número de consultas de pré-natal ${ }^{(3)}$.

No Brasil, a TMI vem diminuindo nas últimas décadas. Tal fato se deve à implantação de programas sociais e de saúde pelo Governo Federal, visando melhor assistência à saúde da mulher e da criança. Exemplo de programas exitosos na redução da MI são o incentivo ao aleitamento materno e a Rede Cegonha $^{(4)}$.

Com o fim da era dos Objetivos de Desenvolvimento do Milênio em 2015, projeções relativas às taxas de mortalidade no país, em especial no período neonatal, apontam que estas ainda permanecerão elevadas variando o seu nível de crescimento de acordo com as causas, dentre as quais se destacam prematuridade, malformação congênita, asfixia intra-parto, infecções perinatais e fatores maternos, com proporção considerável de mortes preveníveis por ação dos serviços de saúde ${ }^{(2,5-6)}$.

Neste sentido, descrever o perfil dos óbitos neonatais e das causas evitáveis no município de Porto Velho, estado de Rondônia, no período de 2011 a 2015, permitirá identificar as fragilidades e desafios existentes, no que concerne a oferta do cuidado às gestantes e neonatos nos serviços de saúde, considerados como referência para o estado e áreas de fronteira.

\section{- MÉTODO}

Trata-se de estudo epidemiológico descritivo, do tipo transversal com base nos dados secundários obtidos do Sistema de Informação de Nascidos Vivos (SINASC) e Sistema de Informação de Mortalidade (SIM), no período de janeiro de 2011 a dezembro de 2015, disponibilizados pela Secretaria Municipal de Saúde de Porto Velho. O período de coleta de dados ocorreu em abril de 2016.

A população do estudo foi composta por todos os óbitos neonatais de gestantes residentes no referido município, informados e registrados na base de dados dos sistemas de informação de saúde supracitados. Como critério de inclusão, foram utilizados todos os óbitos neonatais que tiveram as Declarações de Óbito (DO) preenchidas com $50 \%$ ou mais dos campos das variáveis. E como exclusão, os óbitos que não tinham o registro do ano ou número da Declaração de Nascido Vivo (DNV).

As variáveis selecionadas para o estudo foram: sociodemográficas maternas, epidemiológicas neonatais, obstétricas, característica relacionada à causa básica do óbito e população de recémnascido $(\mathrm{RN})$ para o cálculo do coeficiente de mortalidade neonatal $(\mathrm{CMN})$. Para a obtenção do óbito evitável, a variável causa básica de óbito foi codificada segundo a Classificação Estatística Internacional de Doenças e Problemas Relacionados à Saúde - 10ª Revisão (CID-10). Os óbitos foram classificados em subgrupos de mortes evitáveis, conforme a atualização da lista de causas de mortes evitáveis por intervenções do Sistema Único de Saúde do Brasil( ${ }^{(7)}$, sendo elas: Reduzíveis por atenção à mulher e ao RN; Reduzíveis por adequada atenção à mulher na gestação; Reduzíveis por adequada atenção à mulher no parto; Reduzíveis por adequada atenção ao RN; Reduzíveis por ações adequadas de diagnóstico e tratamento; Causas de morte mal definidas; Demais causas (não claramente evitáveis).

Os dados foram analisados por meio de distribuição de frequência no programa Excel. Este estudo atendeu aos aspectos éticos da Resolução 466/2012(8), conforme aprovado sob parecer $n^{\circ} 870.192$ CEP/ NUSAU/UNIR. 
O número de NV no município no período de 2011 a 2015 foi de 30.236, dos quais 643 foram a óbito no período neonatal e destes 279 foram elegíveis para este estudo. Ressalta-se que 56,61\% (364) dos óbitos neonatais não tiveram as DO preenchidas em pelo menos $50 \%$ dos campos das variáveis.

As mães eram em sua maioria jovens, com ensino médio, do lar, número de consultas de pré-natal inferior ao preconizado pelo Ministério da Saúde (mínimo seis consultas), gestação única, pré termo e via de parto cesárea (Tabela 1).

Tabela 1 - Distribuição das características maternas, segundo variáveis sociodemográficas e obstétricas no período de 2011 a 2015. Porto Velho, RO, Brasil, 2016 (continua)

\begin{tabular}{|c|c|c|c|c|c|c|c|c|c|c|c|c|}
\hline \multirow{2}{*}{$\begin{array}{l}\text { Características } \\
\text { Selecionadas }\end{array}$} & \multicolumn{2}{|c|}{2011} & \multicolumn{2}{|c|}{2012} & \multicolumn{2}{|c|}{2013} & \multicolumn{2}{|c|}{2014} & \multicolumn{2}{|c|}{2015} & \multicolumn{2}{|c|}{ TOTAL } \\
\hline & $\mathbf{N}$ & $\%$ & $\mathbf{N}$ & $\%$ & $\mathbf{N}$ & $\%$ & $\mathbf{N}$ & $\%$ & $\mathbf{N}$ & $\%$ & $\mathbf{N}$ & $\%$ \\
\hline \multicolumn{13}{|l|}{ Idade da mãe } \\
\hline 10 a 19 & 32 & 29,09 & 27 & 24,77 & 8 & 26,67 & 5 & 23,81 & 1 & 11,11 & 73 & 26,16 \\
\hline 20 a 29 & 47 & 42,73 & 59 & 54,13 & 19 & 63,33 & 13 & 61,9 & 5 & 55,56 & 143 & 51,25 \\
\hline 30 a 39 & 27 & 24,55 & 21 & 19,27 & 3 & 10 & 3 & 14,29 & 2 & 22,22 & 56 & 20,07 \\
\hline 40 a 49 & 3 & 2,73 & 2 & 1,83 & - & - & - & - & 1 & 11,11 & 6 & 2,15 \\
\hline Ignorado & 1 & 0,91 & - & & - & - & - & - & - & - & 1 & 0,36 \\
\hline \multicolumn{13}{|l|}{ Escolaridade } \\
\hline Sem escolaridade & 1 & 0,91 & 3 & 2,752 & - & - & - & - & 1 & 11,11 & 5 & 1,79 \\
\hline Ensino fundamental & 18 & 16,36 & 10 & 9,174 & - & - & 2 & 9,524 & 1 & 11,11 & 31 & 11,11 \\
\hline Ensino médio & 83 & 75,45 & 75 & 68,81 & 29 & 96,67 & 14 & 66,67 & 6 & 66,67 & 207 & 74,19 \\
\hline Ensino superior & 8 & 7,27 & 17 & 15,6 & - & - & 2 & 9,52 & 1 & 11,11 & 28 & 10,04 \\
\hline Ignorado & - & - & 4 & 3,67 & 1 & 3,33 & 3 & 14,29 & - & - & 8 & 2,87 \\
\hline \multicolumn{13}{|l|}{ Ocupação } \\
\hline Do lar & 70 & 63,64 & 74 & 67,89 & 18 & 60 & 13 & 61,9 & 7 & 77,78 & 182 & 65,23 \\
\hline Estudante & 2 & 1,82 & 4 & 3,67 & 1 & 3,33 & - & - & 1 & 11,11 & 8 & 2,86 \\
\hline Outros & 25 & 22,73 & 22 & 20,18 & 9 & 30 & 8 & 38,1 & 1 & 11,11 & 65 & 23,3 \\
\hline Ignorado & 13 & 11,82 & 9 & 8,26 & 2 & 6,67 & - & - & - & - & 24 & 8,6 \\
\hline \multicolumn{13}{|c|}{ Número de consultas de pré-natal } \\
\hline Menos de 4 consultas & 44 & 40 & 44 & 40,37 & 4 & 13,33 & 13 & 61,9 & 2 & 22,22 & 107 & 38,35 \\
\hline De 4 a 6 consultas & 27 & 24,55 & 42 & 38,53 & 15 & 50 & 5 & 23,81 & 4 & 44,44 & 93 & 33,33 \\
\hline 7 consultas e mais & 39 & 35,45 & 19 & 17,43 & 9 & 30 & 3 & 14,29 & 1 & 11,11 & 71 & 25,45 \\
\hline Ignorado & - & - & 4 & 3,67 & 2 & 6,66 & - & - & 2 & 22,22 & 8 & 2,86 \\
\hline \multicolumn{13}{|l|}{ Tipo de gravidez } \\
\hline Única & 101 & 91,82 & 99 & 90,83 & 29 & 96,67 & 21 & 100 & 9 & 100 & 259 & 92,83 \\
\hline Múltipla & 7 & 6,36 & 8 & 7,34 & 1 & 3,33 & - & - & - & - & 16 & 5,73 \\
\hline Ignorado & 2 & 1,82 & 2 & 1,83 & - & - & - & - & - & - & 4 & 1,43 \\
\hline \multicolumn{13}{|l|}{ Idade gestacional } \\
\hline $\begin{array}{l}\text { Pré-termo (22 a } 36 \\
\text { semanas) }\end{array}$ & 52 & 47,27 & 61 & 55,96 & 10 & 33,33 & 17 & 80,95 & 7 & 77,78 & 147 & 52,69 \\
\hline $\begin{array}{l}\text { Termo ( } 37 \text { a } 41 \\
\text { semanas) }\end{array}$ & 31 & 28,18 & 24 & 22,02 & 17 & 56,67 & - & - & 2 & 22,22 & 74 & 26,52 \\
\hline $\begin{array}{l}\text { Pós-termo (42 semanas } \\
\text { e mais) }\end{array}$ & - & - & 2 & 1,83 & - & - & - & - & - & - & 2 & 0,72 \\
\hline Ignorado & 27 & 24,55 & 22 & 20,18 & 3 & 10 & 4 & 19,05 & - & - & 56 & 20,07 \\
\hline
\end{tabular}




\begin{tabular}{|c|c|c|c|c|c|c|c|c|c|c|c|c|}
\hline \multicolumn{13}{|c|}{ Via de nascimento } \\
\hline Cesárea & 60 & 54,55 & 66 & 60,55 & 18 & 60 & 6 & 28,57 & 3 & 33,33 & 153 & 54,84 \\
\hline Vaginal & 50 & 45,45 & 35 & 32,11 & 12 & 40 & 15 & 71,43 & 6 & 66,67 & 118 & 42,29 \\
\hline Ignorado & - & - & 8 & 7,33 & - & - & - & - & - & - & 8 & 2,86 \\
\hline
\end{tabular}

Fonte: SINASC/ SIM- Departamento de Epidemiologia/SEMUSA, 2016

Os óbitos neonatais foram em sua maioria precoce, sexo masculino, caracterizados pelo baixo peso ao nascer, Apgar variando de 6 a 10 no primeiro e quinto minuto, sem anomalias (Tabela 2).

Tabela 2 - Distribuição das características do óbito neonatal no período de 2011 a 2015. Porto Velho, RO, Brasil, 2016

\begin{tabular}{|c|c|c|c|c|c|c|c|c|c|c|c|c|}
\hline \multirow{3}{*}{$\begin{array}{l}\text { Características } \\
\text { Selecionadas }\end{array}$} & \multicolumn{12}{|c|}{ Mortalidade Neonatal } \\
\hline & \multicolumn{2}{|c|}{2011} & \multicolumn{2}{|c|}{2012} & \multicolumn{2}{|c|}{2013} & \multicolumn{2}{|c|}{2014} & \multicolumn{2}{|c|}{2015} & \multicolumn{2}{|c|}{ Total } \\
\hline & $\mathbf{N}$ & $\%$ & $\mathbf{N}$ & $\%$ & $\mathbf{N}$ & $\%$ & $\mathbf{N}$ & $\%$ & $\mathbf{N}$ & $\%$ & $\mathbf{N}$ & $\%$ \\
\hline \multicolumn{13}{|c|}{ Classificação do óbito } \\
\hline Precoce & 71 & 64,55 & 89 & 81,65 & 24 & 80 & 16 & 76,19 & 8 & 88,89 & 208 & 74,55 \\
\hline Tardio & 39 & 35,45 & 20 & 18,35 & 6 & 20 & 5 & 23,81 & 1 & 11,11 & 71 & 25,45 \\
\hline \multicolumn{13}{|l|}{ Sexo } \\
\hline Masculino & 68 & 61,82 & 54 & 49,54 & 16 & 53,33 & 14 & 66,67 & 4 & 44,44 & 156 & 55,91 \\
\hline Feminino & 42 & 38,18 & 53 & 48,62 & 14 & 46,67 & 7 & 33,33 & 4 & 44,44 & 120 & 43,01 \\
\hline Ignorado & - & - & 2 & 1,83 & - & - & - & - & 1 & 11,11 & 3 & 1,07 \\
\hline \multicolumn{13}{|l|}{ Peso ao nascer } \\
\hline $\begin{array}{l}\text { Até } 2.499 \\
\text { gramas }\end{array}$ & 60 & 54,55 & 87 & 79,82 & 21 & 70 & 20 & 95,24 & 6 & 66,67 & 194 & 69,53 \\
\hline $\begin{array}{l}2.500 \text { gramas } \\
\text { e mais }\end{array}$ & 27 & 24,55 & 18 & 16,51 & 7 & 23,33 & 1 & 4,76 & 3 & 33,33 & 56 & 20,07 \\
\hline Ignorado & 23 & 20,91 & 4 & 3,67 & 2 & 6,67 & - & - & - & - & 29 & 10,39 \\
\hline \multicolumn{13}{|c|}{ Apgar no $1^{\circ}$ minuto } \\
\hline 0 a 5 & 36 & 32,73 & 39 & 35,78 & 1 & 3,33 & 12 & 57,14 & 3 & 33,33 & 91 & 32,62 \\
\hline 6 a 10 & 67 & 60,91 & 62 & 56,88 & 29 & 96,67 & 8 & 38,1 & 4 & 44,44 & 170 & 60,93 \\
\hline Ignorado & 7 & 6,36 & 8 & 7,34 & - & - & 1 & 4,76 & 2 & 22,22 & 18 & 6,45 \\
\hline \multicolumn{13}{|c|}{ Apgar no $5^{\circ}$ minuto } \\
\hline 0a 5 & 17 & 15,45 & 15 & 13,76 & - & - & 5 & 23,81 & 1 & 11,11 & 38 & 13,62 \\
\hline 6 a 10 & 88 & 80 & 86 & 78,9 & 30 & 100 & 15 & 71,43 & 7 & 77,78 & 226 & 81 \\
\hline Ignorado & 5 & 4,55 & 8 & 7,33 & - & - & 1 & 4,76 & 1 & 11,11 & 15 & 5,38 \\
\hline \multicolumn{13}{|l|}{ Anomalias } \\
\hline Não & 105 & 95,45 & 101 & 92,66 & 29 & 96,67 & 19 & 90,48 & 7 & 77,78 & 261 & 93,55 \\
\hline Sim & 5 & 4,55 & 8 & 7,34 & 1 & 3,33 & 2 & 9,52 & 2 & 22,22 & 18 & 6,45 \\
\hline
\end{tabular}

Fonte: SINASC/ SIM- Departamento de Epidemiologia/SEMUSA, 2016

Durante o período de 2011 a 2013, o município de Porto Velho apresentou um CMN crescente, mas com diminuição abrupta em 2014 e 2015 (Tabela 3). 
Tabela 3 - Distribuição dos óbitos neonatais, nascidos vivos e coeficiente de mortalidade neonatal no período de 2011 a 2015. Porto Velho, RO, Brasil, 2016

\begin{tabular}{|c|c|c|c|c|c|c|}
\hline \multirow[t]{2}{*}{ Ano } & \multicolumn{2}{|c|}{ Óbitos Neonatais } & \multicolumn{2}{|c|}{ Nascidos Vivos } & \multirow[t]{2}{*}{ CMN* } & \multirow{2}{*}{$\begin{array}{l}\text { CMN* } \\
\text { Precoce }\end{array}$} \\
\hline & $\mathbf{N}$ & $\%$ & $\mathbf{N}$ & $\%$ & & \\
\hline 2011 & 110 & 39,43 & 9773 & 32,32 & 11,25 & 7,26 \\
\hline 2012 & 109 & 39,07 & 9252 & 30,6 & 11,78 & 9,62 \\
\hline 2013 & 30 & 10,75 & 1742 & 5,76 & 17,22 & 13,78 \\
\hline 2014 & 21 & 7,53 & 5538 & 18,32 & 3,79 & 2,89 \\
\hline 2015 & 9 & 3,23 & 3931 & 13 & 2,28 & 2,04 \\
\hline Total & 279 & 100 & 30236 & 100 & 9,23 & 6,88 \\
\hline
\end{tabular}

*Coeficiente de Mortalidade Neonatal

Fonte: SINASC/ SIM- Departamento de Epidemiologia/SEMUSA, 2016

Chama a atenção em Porto Velho o fato de os óbitos neonatais, entre 2013 e 2015, tenha brusca redução, mesmo com a discreta melhoria das condições sociais e de saúde dessa Unidade Federada. Esses dados são sugestivos de subnotificações, o que caracteriza situação crítica, pois compromete análises epidemiológicas fidedignas. Considerando que a maioria dos óbitos neonatais precoces ocorre em ambientes hospitalares, era de se esperar maior confiabilidade das informações nas DO.

A principal causa básica de óbito foi relacionada à inadequação aos cuidados prestados ao RN, na qual se destaca a septicemia bacteriana não especificada do RN, seguida por causas evitáveis por adequada atenção à mulher na gestação, principalmente por meio da síndrome da angústia respiratória do RN (Tabela 4).

Tabela 4 - Distribuição das causas nos óbitos neonatais no período de 2011 a 2015, segundo a Classificação Internacional de Doenças - CID e a classificação de evitabilidade. Porto Velho, RO, Brasil, 2016 (continua)

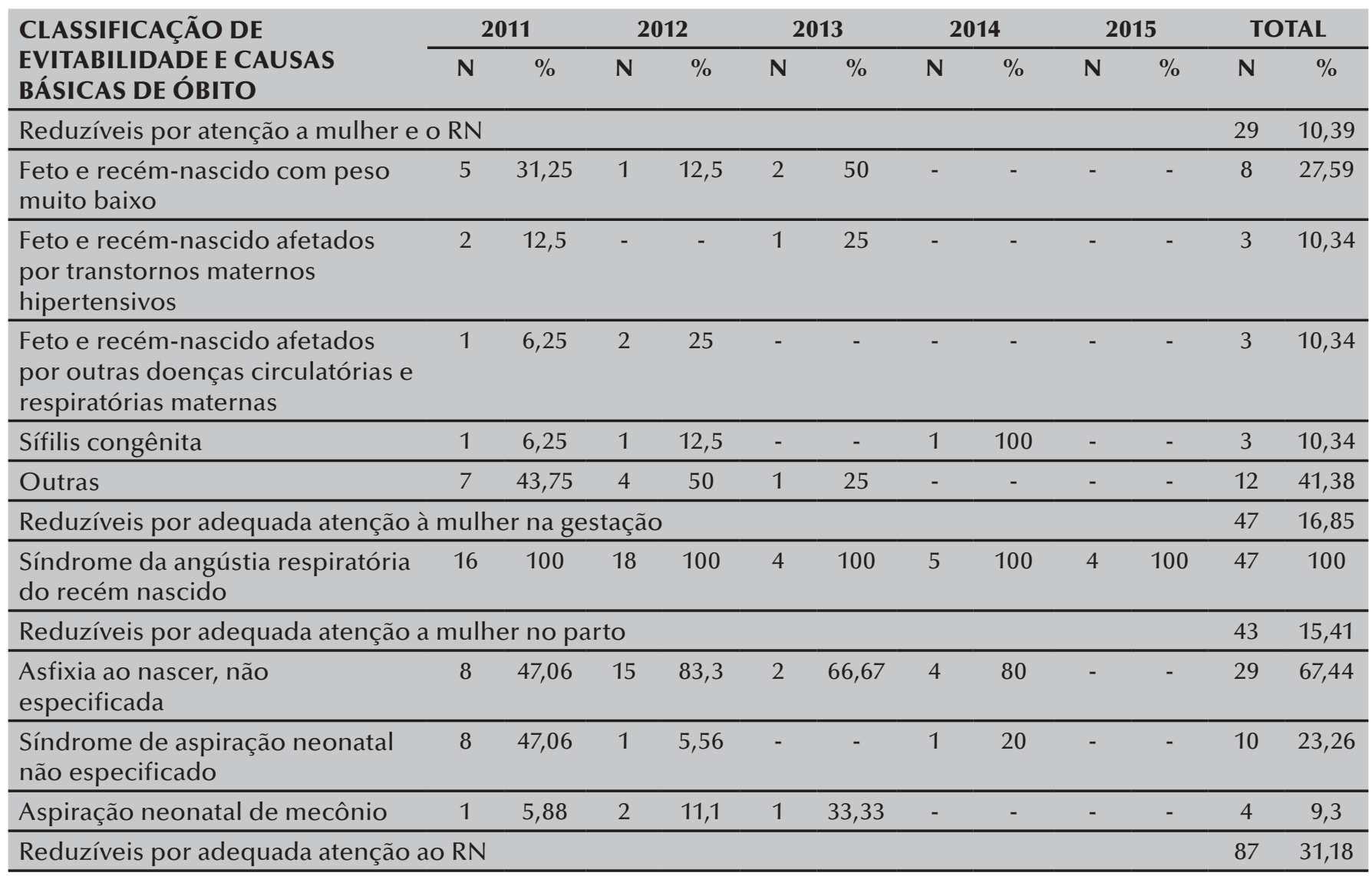


Cogitare Enferm. (22)2: e48950, 2017

\begin{tabular}{|c|c|c|c|c|c|c|c|c|c|c|c|c|}
\hline $\begin{array}{l}\text { Septicemia bacteriana não } \\
\text { especificado do recém-nascido }\end{array}$ & 15 & 53,57 & 18 & 75 & 6 & 50 & 4 & 57,14 & 3 & 100 & 46 & 52,87 \\
\hline $\begin{array}{l}\text { Atelectasia primária do recém } \\
\text { nascido }\end{array}$ & 2 & 7,14 & 6 & 25 & 2 & 16,67 & 1 & 14,29 & - & - & 11 & 12,64 \\
\hline Outras & 11 & 39,29 & 13 & 31 & 4 & 33,33 & 2 & 28,57 & - & - & 30 & 34,48 \\
\hline \multicolumn{11}{|c|}{ Reduzíveis por ações adequadas de diagnóstico e tratamento } & 2 & 0,72 \\
\hline Meningite & 1 & 100 & - & - & - & - & 1 & 100 & - & - & 2 & 100 \\
\hline Causas de morte mal definidas & & & & & & & & & & & 2 & 0,72 \\
\hline $\begin{array}{l}\text { Afecções originadas no período } \\
\text { perinatal não especificadas }\end{array}$ & 1 & - & - & - & - & - & 1 & - & - & - & 2 & 100 \\
\hline \multicolumn{11}{|c|}{ Demais causas (não claramente evitáveis) } & 69 & 24,73 \\
\hline Anencefalia & 4 & 12,9 & 5 & 17,9 & 1 & 16,67 & 1 & 50 & 1 & 50 & 12 & 17,39 \\
\hline Atelectasia & 3 & 9,68 & 4 & 14,3 & 2 & 33,33 & - & - & - & - & 9 & 13,04 \\
\hline $\begin{array}{l}\text { Malformações congênitas } \\
\text { múltiplas, não classificadas em } \\
\text { outra parte }\end{array}$ & 3 & 9,68 & 3 & 10,7 & - & - & 1 & 50 & 1 & 50 & 8 & 11,59 \\
\hline Outras & 21 & 67,74 & 16 & 57,1 & 3 & 50 & - & - & - & - & 40 & 57,97 \\
\hline
\end{tabular}

Fonte: SINASC/ SIM- Departamento de Epidemiologia/SEMUSA, 2016

\section{- DISCUSSÃO}

Os óbitos neonatais ocorreram predominantemente nos filhos de mulheres jovens entre 20 e 29 anos $(51,25 \%)$, seguindo a tendência de que a mortalidade neonatal ocorre em mulheres com idade inferior a $34 \operatorname{anos}^{(9-10)}$ em razão da maior taxa de fecundidade nesta faixa etária e maior vulnerabilidade social.

Os resultados dessa pesquisa estão em discordância com outros estudos, em que a mortalidade neonatal também tem sido associada aos determinantes distais socioeconômicos, entre eles o baixo nível de escolaridade materna, fator determinante da mortalidade infantil muito enfatizado na literatura, em que filhos nascidos de mulheres com baixa escolaridade têm maior chance de morrer antes de completarem o primeiro ano de $\operatorname{vida}^{(11-12)}$.

O número de consultas de pré-natal nesse estudo foi inferior a quatro (38,35\%) e sabe-se que garantir pelo menos seis consultas de pré-natal com o início antes de 12 semanas beneficia as gestantes, independente de idade, classe social ou nível instrucional, uma vez que viabiliza acesso ao diagnóstico precoce de possíveis patologias na gestação que possam ocasionar prejuízo na saúde da mulher e da criança, além de proporcionar um melhor acompanhamento do desenvolvimento do bebê $\hat{e}^{(13)}$.

Estudos afirmam que indicadores socioeconômicos, entre eles, trabalhar fora - a grande maioria de mulheres nesta pesquisa era do lar - representa fator de risco para a mortalidade neonatal ${ }^{(14-15)}$, tendo em vista que a disponibilidade de tempo das mães em realizar as consultas de pré-natal e o autocuidado se torna restrita, afetando diretamente a qualidade de vida do neonato ${ }^{(15)}$.

Apesar de o estudo ter apresentado maiores óbitos na gravidez com feto único (92,83\%), ainda que este se caracterize como fator de proteção para o $\mathrm{RN}^{(16)}$, salienta-se que existe forte associação da morbiletalidade neonatal com as gestações múltiplas, por ser fator de risco para a prematuridade e o crescimento intra-uterino ${ }^{(17)}$.

A via de nascimento predominante em Porto Velho correspondeu a cesariana (54,84\%), cujos achados se equivalem aos estudos em que $56,7 \%^{\left({ }^{(6)}\right.}$ e $52 \%{ }^{(18)}$ dos óbitos neonatais foram de mães submetidas à cesárea.

Verificou-se que houve menor percentual de óbitos com o parto vaginal (42,29\%), que contribui com o fortalecimento do sistema imunológico, o desenvolvimento do ritmo cardíaco e maturação 
pulmonar do RN. O parto vaginal é fisiológico e o corpo da mulher foi preparado para tal evento ${ }^{(18)}$. Além disso, um estudo realizado no Rio Grande do Sul relaciona a prematuridade como causa de parto cesáreo pela margem de erro na idade gestacional ${ }^{(19)}$.

Os resultados encontrados quanto ao sexo masculino dos óbitos neonatais corroboram com estudo realizado em Fortaleza ${ }^{(14)}$. Esta diferença entre os sexos ocorre desde o período intra-uterino. $\mathrm{O}$ sexo feminino apresenta melhor adaptação metabólica aos estressores gestacionais, proporcionando melhor adaptação no nascimento, e consequentemente menor mortalidade ${ }^{(20)}$.

Este estudo revela discrepância crescente de óbitos prematuros, principalmente após baixo percentual em 2013 (33,33\%) quando comparado com os anos de 2014 e 2015 (80,95\% e 77,78\% respectivamente), o que pode ser resultado das induções cesarianas ${ }^{(21)}$. Sabe-se que o óbito neonatal é predominante em RNs com baixo peso e prematuros ${ }^{(6)}$.

Uma das limitações desse estudo refere-se à análise dos óbitos no SIM nos anos 2013, 2014 e 2015, evidenciando-se baixo registro de óbitos neonatais, o que prejudicou análises mais fidedignas sobre a mortalidade neonatal em Porto Velho. Afinal, este indicador caracteriza a qualidade dos serviços de saúde, saneamento básico e educação. O SINASC também apresentou baixo registro de NV em 2013. Tal fato não comprometeu as informações disponibilizadas para caracterizar a mortalidade neonatal, as quais geralmente estão associadas a fatores biológicos, assistenciais e socioeconômicos.

Observou-se predominância do Apgar entre 6 e 10 , tanto no $1^{\circ}$ minuto $(60,93 \%)$ como no $5^{\circ}$ minuto (81\%). Entretanto estudos realizados no Ceará e no Rio Grande do Sul demonstraram que índices de Apgar menores ou iguais a oito são preditivos ao risco de morte, colocando o RN em situação de maior vulnerabilidade ${ }^{(14,22)}$.

Neste estudo, não houve anomalias em 93,55\% dos óbitos, embora tais condições possam aumentar o desfecho para mortalidade neonatal ${ }^{(23)}$.

O CMN estudado foi de 9,23/1000NV, sendo este inferior aos dados encontrados em Recife-PE $(10,99 / 1000 N V)^{(24)}$. No entanto, quando comparado a outros estudos, esse coeficiente torna-se superior, uma vez que em Cuiabá-MT o CMN encontrado foi de 8,2 $2^{(25)}$ e no estado do Rio Grande do Sul 8,3 ${ }^{(22)}$.

O CMNP em Porto Velho foi de 6,88, semelhante ao encontrado em outro estudo realizado em Cuiabá-MT (6,0/1000NV) ${ }^{(25)}$. Tal aspecto está associado às fragilidades da atenção durante o prénatal, assistência ao RN e falta de acesso à informação. Esse achado não sugere decréscimo real da mortalidade neonatal, tendo em vista o elevado índice de sub-registros, principalmente nos estados do Norte e Nordeste, o que demanda cautela na análise dos dados ${ }^{(26)}$. É indispensável sistemas de informação confiáveis para análises de mortalidade, haja vista que as estimativas do coeficiente de óbitos neonatais são complexas tanto no numerador (óbitos neonatais) quanto no denominador (NV).

Dentre as principais causas de óbitos em Porto Velho, 31,18\% são reduzíveis por adequada atenção ao $\mathrm{RN}$, bem como à mulher na gestação $(16,85 \%)$ e no parto $(15,41 \%)$. Um estudo realizado na região central do estado de Minas Gerais descreveu que a evolução da MI evitável apresentou resultados semelhantes à realidade de Porto Velho, sendo 36,5\% referentes à atenção ao RN e 28,1\% à mulher na gestação ${ }^{(27)}$.

No estudo realizado em Cuiabá, achados semelhantes foram encontrados, sendo a maior proporção dos óbitos reduzíveis por adequada atenção ao $\mathrm{RN}^{(25)}$. Quanto às causas reduzíveis por adequada atenção da mulher no parto, a asfixia ao nascer foi a principal causa $(31,5 \%)$ em um estudo realizado em Salvador ${ }^{(28)}$ se assemelhando aos resultados encontrados neste estudo $(67,44 \%)$.

É importante destacar que um pré-natal de qualidade está diretamente relacionado ao bemestar materno-infantil, pois o acompanhamento não adequado durante o pré-natal acarreta graves consequências ao binômio mãe-filho, como por exemplo, infecções do trato geniturinário recorrentes, prematuridade e o baixo peso ao nascer, sendo estes os principais fatores de risco para a sepse neonatal ${ }^{(29)}$.

Além disso, é necessário melhorar a qualidade da assistência em saúde, bem como o diagnóstico e tratamento precoce das infecções ocorridas durante pré-natal, parto e nascimento, uma vez que os resultados encontrados foram preocupantes e poderiam ser evitados, por meio de serviços de saúde 
efetivos com medidas de prevenção e tratamento do fenômeno ou da condição que o determina ${ }^{(30)}$.

A redução da mortalidade neonatal está associada à importância que o gestor municipal, serviços, sistema de saúde e sociedade atribuem a estas altas taxas de mortalidade, principalmente nos primeiros seis dias de $v_{i d a}{ }^{(6)}$.

Os resultados sugerem que as informações declaradas no SINASC e SIM ainda apresentam muitas variáveis ignoradas ou incompletas, o que prejudica a sua qualidade. Provavelmente, esses dados preenchidos de forma não adequada nas DO e DNV devem-se à inexistência dos comitês de óbito infantil e fetal em Porto Velho, os quais contribuiriam para a qualificação da informação, além de sensibilizar profissionais para o registro adequado das estatísticas vitais.

Outro fator que pode estar relacionado a esta realidade é a localização do município de estudo, que possui uma grande extensão de áreas ribeirinhas e rurais, com enormes vazios assistenciais e de serviços que dificultam os fluxos de informação e comunicação, favorecendo as subnotificações e os sub-registros dos sistemas de informação em saúde. Tal fato dificulta a construção dos indicadores para a organização e gestão das redes de atenção à saúde.

\section{CONCLUSÃO}

Neste estudo prevaleceram os óbitos precoces, do sexo masculino, com peso até 2.499 gramas, Apgar variando de 6 a 10 no $1^{\circ}$ minuto e $5^{\circ}$ minuto, sem anomalia. O coeficiente de mortalidade neonatal foi crescente com diminuição abrupta nos anos de 2014 e 2015. A causa básica predominante foi reduzível por adequada atenção ao recém-nascido. Tais resultados alertam para necessidade de melhoria na assistência pré-natal e estruturação da rede cegonha no município de modo a garantir uma atenção de qualidade, integrada e contínua ao binômio mãe/filho.

A falta do preenchimento nos campos da DO e DNV dificultou a realização de uma análise fidedigna de alguns achados da pesquisa. Para isto, é necessário sensibilizar o profissional e capacitá-lo sobre a importância do preenchimento correto das fichas para a geração de informações adequadas que possam mudar essa realidade. É fundamental que o profissional saiba do seu papel na construção dos indicadores de qualidade, pois são eles que subsidiam o planejamento de ações e de políticas de saúde eficazes. Além, de sua participação na mudança desses resultados.

\section{REFERÊNCIAS}

1. dos Santos HG, de Andrade SM, Silva AMR, Mathias TAF, Ferrari LL, Mesas AE. Mortes infantis evitáveis por intervenções do Sistema Único de Saúde: comparação de duas coortes de nascimentos. Ciênc. saúde coletiva. [Internet] 2014;19(3) [acesso em 03 fev 2016]. Disponível: http://dx.doi.org/10.1590/1413-81232014193.01182013.

2. Liu L, Oza S, Hogan D, Perin J, Rudan I, Lawn JE, et al. Global, regional, and national causes of child mortality in 2000-13, with projections to inform post-2015 priorities: an updated systematic analysis. The Lancet. [Internet] 2015;385(9966) [acesso em 25 jan 2017]. Disponível: http://dx.doi.org/10.1016/S0140-6736(14)61698-6.

3. United Nations Children's Fund (Unicef). Levels \& Trends in Child Mortality. Estimates Developed by the UN Inter-agency Group for Child Mortality Estimation. [Internet] 2015 [acesso em 14 mar 2017]. Disponível: https:// www.unicef.org/publications/files/Child_Mortality_Report_2015_Web_8_Sept_15.pdf

4. Ministério da Saúde (BR). Mortalidade Infantil no Brasil: tendências, componentes e causas de morte no período de 2000 a 2010. In: Saúde Brasil 2011: uma análise da situação de saúde e a vigilância da saúde da mulher. [Internet] Brasília: Ministério da Saúde; 2012 [acesso em 11 mar 2017]. Disponível: http://repositorio.unb.br/ bitstream/10482/12478/1/CAPITULO_MortalidadelnfantilBrasil.pdf.

5. Liu L, Oza S, Hogan D, Chu Y, Perin J, Zhu J, et al. Global, regional, and national causes of under-5 mortality in 2000-15: an updated systematic analysis with implications for the Sustainable Development Goals. The Lancet. [Internet] 2017;388(10063) [acesso em 25 jan 2017]. Disponível: http://dx.doi.org/10.1016/S0140-6736(16)31593-8.

6. Lansky S, Friche AAL, da Silva AAM, Campos D, Bittencourt SDA, de Carvalho ML, et al. Pesquisa Nascer no 
Brasil: perfil da mortalidade neonatal e avaliação da assistência à gestante e ao recém-nascido. Cad. Saúde Pública. [Internet] 2014;30(Supl 1) [acesso em 12 jun 2016]. Disponível: http://dx.doi.org/10.1590/0102-311X00133213.

7. Malta DC, Sardinha LMV, de Moura L, Lansky S, Leal MC, Szwarcwald CL, et al. Atualização da lista de causas de mortes evitáveis por intervenções do Sistema Único de Saúde do Brasil. Epidemiol. Serv. Saúde [Internet] 2010;19(2) [acesso em 3 fev 2016]. Disponível: http://scielo.iec.pa.gov.br/pdf/ess/v19n2/v19n2a10.pdf.

8. Ministério da Saúde (BR). Conselho Nacional de Saúde. Diretrizes e normas regulamentadoras de pesquisa envolvendo seres humanos. Resolução n. 466, de 12 de dezembro de 2012. Brasília; 2012.

9. Ortiz LP, Oushiro DA. Perfil da mortalidade neonatal no estado de São Paulo. São Paulo em Perspectiva. [Internet] 2008;22(1) [acesso em 12 mar 2016]. Disponível: http://produtos.seade.gov.br/produtos/spp/v22n01/ v22n01_02.pdf.

10. Soares ES, Menezes GMS. Fatores associados à mortalidade neonatal precoce: análise de situação no nível local. Epidemiol. Serv. Saúde. [Internet] 2010;19(1) [acesso em 11 mar 2017]. Disponível: http://dx.doi.org/10.5123/ S1679-49742010000100007.

11. Jobim R, Aerts D. Mortalidade infantil evitável e fatores associados em Porto Alegre, Rio Grande do Sul, Brasil, 2000-2003. Cad. Saúde Pública. [Internet] 2008;24(1) [acesso em 11 mar 2017]. Disponível: http://dx.doi. org/10.1590/S0102-311X2008000100018.

12. Garcia LP, Santana LR. Evolução das desigualdades socioeconômicas na mortalidade infantil no Brasil, 19932008. Ciênc. Saúde Coletiva. [Internet] 2011;16(9) [acesso em 11 mar 2017]. Disponível: http://dx.doi.org/10.1590/ S1413-81232011001000009.

13. Ministério da Saúde (BR). Secretaria de Atenção à Saúde. Departamento de Atenção Básica. Atenção ao PréNatal de Baixo Risco. Série A: Normas e Manuais Técnicos. Caderno de atenção básica nº 32. Brasília: Ministério da Saúde; 2012.

14. Helena ETS, de Sousa CA, da Silva CA. Fatores de risco para mortalidade neonatal em Blumenau, Santa Catarina: linkage entre bancos de dados. Rev. Bras. Saúde Mater. Infant. [Internet] 2005;5(2) [acesso em 12 mar 2016]. Disponível: http://dx.doi.org/10.1590/S1519-38292005000200010.

15. do Nascimento RM. Determinantes da mortalidade neonatal em Fortaleza-CE: um estudo caso controle [dissertação]. Fortaleza (CE): Universidade Federal do Ceará; 2011.

16. de Aguiar JB. Fatores de risco para a mortalidade neonatal, em hospital de referência [dissertação]. Fortaleza (CE): Universidade Estadual do Ceará; 2011.

17. Ministério da Saúde (BR). Secretaria de Atenção à Saúde. Departamento de Ações Programáticas Estratégicas. Gestação de alto risco: manual técnico. $5^{a}$ ed. [Internet] Brasília: Ministério da Saúde; 2012 [acesso em 11 mar 2017]. Disponível: http://bvsms.saude.gov.br/bvs/publicacoes/manual_tecnico_gestacao_alto_risco.pdf.

18. Gaíva MAM, Bittencourt RM, Fujimori E. Óbito neonatal precoce e tardio: perfil das mães e dos recémnascidos. Rev. Gaúcha Enferm. [Internet] 2013;34(4) [acesso em 24 fev 2016]. Disponível: http://dx.doi.org/10.1590/ S1983-14472013000400012.

19. Ministério da Saúde (BR). Secretaria de Atenção à Saúde. Departamento de Ações Programáticas Estratégicas. Manual de Vigilância do Óbito Infantil e Fetal e do Comitê de Prevenção do Óbito Infantil e Fetal. [Internet] Brasília: Ministério da Saúde; 2009 [acesso em 23 jul 2016]. Disponível: http://svs.aids.gov.br/download/manuais/ manual_infantil_fetal03_fim2_1.pdf.

20. da Silva TH, Fensterseifer LM. Prematuridade dos recém-nascidos em Porto Alegre e seus fatores associados. Revista Brasileira de História \& Ciências Sociais - RBHCS. [Internet] 2015;7(13) [acesso em 20 fev 2017]. Disponível: http://dx.doi.org/10.14295/rbhcs.v7i13.305.

21. Misra DP, Salafia CM, Miller RK, Charles AK. Non-linear and gender-specific relationships among placental growth measures and the fetoplacental weight ratio. Placenta. [Internet] 2009;30(12) [acesso em 24 mar 2017]. Disponível: http://dx.doi.org/10.1016/j.placenta.2009.09.008.

22. Victora CG, Aquino EML, Leal MC, Monteiro CA, Barros FC, Szwarcwald CL. Saúde de mães e crianças no Brasil: progressos e desafios. The Lancet. [Internet] 2011; (377(9780) [acesso em 10 jun 2016]. Disponível: http:// 
dx.doi.org/10.1016/S0140-6736(11)60138-4.

23. Zanini RR, de Moraes AB, Giugliani ERJ, Riboldi J. Determinantes contextuais da mortalidade neonatal no Rio Grande do Sul por dois modelos de análise. Rev. Saúde Pública. [Internet] 2011;45(1) [acesso em 13 abr 2016]. Disponível: http://dx.doi.org/10.1590/S0034-89102011000100009.

24. Borba GG, Nevez ET, Arrué AM, da Silveira A, Zamberlan KC. Fatores associados à morbimortalidade neonatal: um estudo de revisão. Rev. Saúde (Santa Maria). [Internet] 2014;40(1) [acesso em 15 mai 2016]. Disponível: http:// dx.doi.org/10.5902/223658347774.

25. Rocha R, Oliveira C, da Silva DKF, Bonfim C. Mortalidade neonatal e evitabilidade: uma análise do perfil epidemiológico. Rev. enferm. UERJ. [Internet] 2011;19(1) [acesso em 10 jun 2016]. Disponível: http://www.facenf. uerj.br/v19n1/v19n1a19.pdf.

26. Lourenço EC, Brunken GS, Luppi CG. Mortalidade infantil neonatal: estudo das causas evitáveis em Cuiabá, Mato Grosso, 2007. Epidemiol. Serv. Saúde. [Internet] 2013;22(4) [acesso em 10 jun 2016]. Disponível: http://scielo. iec.pa.gov.br/pdf/ess/v22n4/v22n4a16.pdf.

27. Lisboa L, de Abreu DMX, Lana AMQ, França EB. Mortalidade infantil: principais causas evitáveis na região Centro de Minas Gerais, Brasil, 1999-2011. Epidemiol. Serv. Saúde. [Internet] 2015;24(4) [acesso em 17 jun 2016]. Disponível: http://dx.doi.org/10.5123/S1679-49742015000400013.

28. Nascimento RCS. Mortalidade perinatal em Salvador: análise espacial das condições de evitabilidade e desigualdades sociais [tese]. Salvador (BA): Instituto de Saúde Coletiva. Universidade Federal da Bahia; 2014.

29. Pires SA, de Almeida NMS. Mortalidade por septicemia bacteriana: um estudo descritivo no período perinatal. Revista Enfermagem Contemporânea. [Internet] 2016;5(1) [acesso em 12 mar 2017]. Disponível: http://dx.doi. org/10.17267/2317-3378rec.v5i1.946.

30. do Nascimento SG, de Oliveira CM, Sposito V, Ferreira DKS, do Bonfim CV. Mortalidade infantil por causas evitáveis em uma cidade do Nordeste do Brasil. Rev. bras. enferm. [Internet] 2014;67(2) [acesso em 10 mar 2017]. Disponível: http://dx.doi.org/10.5935/0034-7167.20140027. 